\title{
Universal amplitudes in the FSS of three-dimensional spin models
}

\author{
Martin Weigelt and Wolfhard Janket \\ Institut für Theoretische Physik, Universität Leipzig, 04109 Leipzig, Germany, and \\ Institut für Physik, Johannes Gutenberg-Universität Mainz, 55099 Mainz, Germany
}

(February 24, 2018)

In a MC study using a cluster update algorithm we investigate the finite-size scaling (FSS) of the correlation lengths of several representatives of the class of three-dimensional classical $\mathrm{O}(n)$ symmetric spin models on the geometry $T^{2} \times \mathbb{R}$. For all considered models we find strong evidence for a linear relation between FSS amplitudes and scaling dimensions when applying antiperiodic instead of periodic boundary conditions across the torus. The considered type of scaling relation can be proven analytically for systems on two-dimensional strips with periodic bc using conformal field theory.

PACS numbers: 64.60.Fr, 75.10.Mk, 75.40.Mg, 11.25.Hf

Conformal invariance of $2 \mathrm{D}$ systems at a critical point has turned out to be the key feature for a complete, analytical description of their critical behavior [1.22. In particular, conformal field theory (CFT) supplies exact FSS relations including the amplitudes for these 2D models. For strips of width $L$ with periodic boundary conditions, i.e. the $S^{1} \times \mathbb{R}$ geometry, Cardy [3] has shown that the FSS amplitudes of the correlation lengths $\xi_{i}$ of primary (conformally covariant) operators are entirely determined by the corresponding scaling dimensions $x_{i}$ :

$$
\xi_{i}=\frac{A}{x_{i}} L
$$

with a model independent overall amplitude $A=1 / 2 \pi$. This result relies on both, the greater restrictive strength of the 2D conformal group compared with the higher dimensional cases, which is needed for the definition of the "primarity" of operators, and the fact that the considered geometry is conformally related to the corresponding flat space $\mathbb{R}^{2}$.

Generalizing these results to more realistic 3D geometries within the CFT framework generically destroys the rich 2D group structure. Keeping at least the conformal flatness condition, Cardy [4] arrived at a conjecture of the form (1) for the $S^{n-1} \times \mathbb{R}, n>2$ geometries. Mainly for reasons of the numerical inaccessibility of these geometries Henkel [5.60] considered the situation where even this latter condition is cancelled: investigating the scaling behavior of the $S=\frac{1}{2}$ Ising model on 3D columns $T^{2} \times \mathbb{R}$ with periodic (pbc) or antiperiodic (apbc) boundary conditions across the torus via a transfer matrix calculation, he found for the correlation lengths of the magnetization and energy densities (the only primary operators in the $2 D$ model) in the scaling regime the ratios:

$$
\begin{array}{ll}
\xi_{\sigma} / \xi_{\epsilon}=3.62(7) & \text { periodic bc, } \\
\xi_{\sigma} / \xi_{\epsilon}=2.76(4) & \text { antiperiodic bc. }
\end{array}
$$

Comparing this to the ratio of scaling dimensions of $x_{\epsilon} / x_{\sigma}=2.7326(16)$ a relation of the form (11) seems not to hold, unless the boundary conditions are changed to be antiperiodic. This is in qualitative agreement with numerical work done by Weston [7].

In this letter, we first revisit the Ising model on the $T^{2} \times \mathbb{R}$ geometry trying to decide the exposed question with an independent Monte Carlo (MC) method and at an increased level of accuracy. The main purpose is to investigate further models - in our case $\mathrm{O}(n), n>1$ spin models -, thus adding evidence that Henkel's result is not just a numerical "accident" but reflects a universal property of such 3D systems.

The model - We consider an $\mathrm{O}(n)$ symmetric classical spin model with nearest-neighbor, ferromagnetic interactions in zero field with Hamiltonian

$$
\mathcal{H}=-J \sum_{<i j>} \mathbf{s}_{i} \cdot \mathbf{s}_{j}, \quad \mathbf{s}_{i} \in S^{n-1} .
$$

The spins are located on a sc lattice of dimensions $\left(L_{x}, L_{y}, L_{z}\right)$ with $L_{x}=L_{y}$, modeling the $T^{2}$ geometry by applying periodic or antiperiodic bc along the $x$ - and $y$-directions. Effects of the finite length of the lattice in the $z$-direction are minimized by choosing $L_{z}$ such that $L_{z} / \xi \gg 1$ and sticking the ends together via periodic bc. As is well known [8], all of these models undergo a continuous phase transition in three dimensions, so that at the critical point the correlation length diverges linearly with the finite length $L=L_{x}$. Particular representatives of this class are the Ising $(n=1)$, the XY $(n=2)$ and the Heisenberg $(n=3)$ model.

The simulation - For our MC simulations we used the Wolff single-cluster update algorithm [9] which is known to be more effective than the Swendsen-Wang [10] update for three-dimensional systems [11]. As we want to consider antiperiodic bc for all systems in addition to the generic periodic bc case, the algorithm had to be adapted to this situation using the fact that in the case of nearest-neighbor interactions antiperiodic bc are equivalent to the insertion of a seam of antiferromagnetic bonds along the relevant boundary.

The primary observables to measure are the connected correlation functions of the spin and the energy density:

$$
\begin{aligned}
& G_{\sigma}^{c}\left(\mathbf{x}_{1}, \mathbf{x}_{2}\right)=\left\langle\mathbf{s}\left(\mathbf{x}_{1}\right) \cdot \mathbf{s}\left(\mathbf{x}_{2}\right)\right\rangle-\langle\mathbf{s}\rangle\langle\mathbf{s}\rangle, \\
& G_{\epsilon}^{c}\left(\mathbf{x}_{1}, \mathbf{x}_{2}\right)=\left\langle\epsilon\left(\mathbf{x}_{1}\right) \epsilon\left(\mathbf{x}_{2}\right)\right\rangle-\langle\epsilon\rangle\langle\epsilon\rangle .
\end{aligned}
$$


The correlation lengths $\xi_{i}$ in Eq. (1) being understood as measuring the correlations in the longitudinal $\mathbb{R}$ direction, one may average over estimates $\hat{G}^{c}\left(\mathbf{x}_{1}, \mathbf{x}_{2}\right)$ such that $\left(\mathbf{x}_{1}-\mathbf{x}_{2}\right) \| \hat{e}_{z}$ and $i \equiv\left|\mathbf{x}_{1}-\mathbf{x}_{2}\right|=$ const, thus ending up at estimates $\hat{G}^{c, \|}(i)$. This average can be improved by considering a zero momentum mode projection [12], i.e., by correlating layer variables made up out of the sum of variables in a given layer $z=$ const instead of the original spins or local energies; this reduces the variance by a factor of $1 / L_{x}^{2}$, the influence of transversal correlations being irrelevant for large distances $i$ [13.

Assuming an exponential long-distance behavior of the correlation functions (4), extracting the correlation lengths via a straightforward fitting procedure requires a nonlinear three-parameter fit of the form

$$
G^{c, \|}(i)=G^{c, \|}(0) \exp (-i / \xi)+\text { const }
$$

since any numerical estimation of $G^{c, \|}(i)$ necessarily fails to reproduce the correct long distance limit $G^{c, \|}(i) \rightarrow 0$ as $i \rightarrow \infty$ exactly. As this amounts to an investment of the gathered statistics into the determination of three parameters, two of which are completely irrelevant for our ends, we used an alternative method which intrinsically eliminates the two irrelevant parameters by using differences and ratios of $\hat{G}^{c, \|}(i)$ rather than the values themselves. Given the correlation function behaves as (5), estimators $\hat{\xi}_{i}$ for the correlation length are given by:

$$
\hat{\xi}_{i}=\Delta\left[\ln \frac{\hat{G}^{c, \|}(i)-\hat{G}^{c, \|}(i-\Delta)}{\hat{G}^{c, \|}(i+\Delta)-\hat{G}^{c, \|}(i)}\right]^{-1} .
$$

The generic value for $\Delta$ is one, but it might be advantageous to choose $\Delta>1$ in order to enhance the local drop of $G^{c, \|}(i)$ between $i$ and $i+\Delta$ (the signal) against the fluctuations (the noise). Following this procedure one ends up with a set of estimators for the correlation length as a function of distance $i$ as depicted in Fig. 1 for the spin-spin correlations of the Ising model: after a transition regime starting at $i=\Delta$ which is a consequence of the discreteness of the lattice as well as the above mentioned zero momentum mode projection, the estimates settle at a plateau indicating that the exponential long distance behavior has been reached.

The error bars in Fig. 1 were generated using a combined binning and "jackknife" resampling scheme [14 15. which is necessary due to the strong non-linearity of the transformation (6); on the same grounds we checked for the necessity of a bias correction. Final values for the correlation lengths were obtained by an average of the estimators $\hat{\xi}_{i}$; as neither the estimates for very small distances $i$ nor - because of the periodicity of the lattice in the $z$-direction - those for distances $i \gtrsim L_{z} / 2$ are reliable estimates for the continuum correlation length, the range of distances $i_{\min }, \ldots, i_{\max }$ to average over was determined by a procedure of statistical optimization, a generalized $\chi^{2}$-test. In order to minimize the theoretical variance of the final average $\bar{\xi}$ each element $\hat{\xi}_{i}$ was weighted by a factor proportional to a row sum of the inverse covariance matrix, this matrix itself being again estimated by a jackknife technique [13,16.

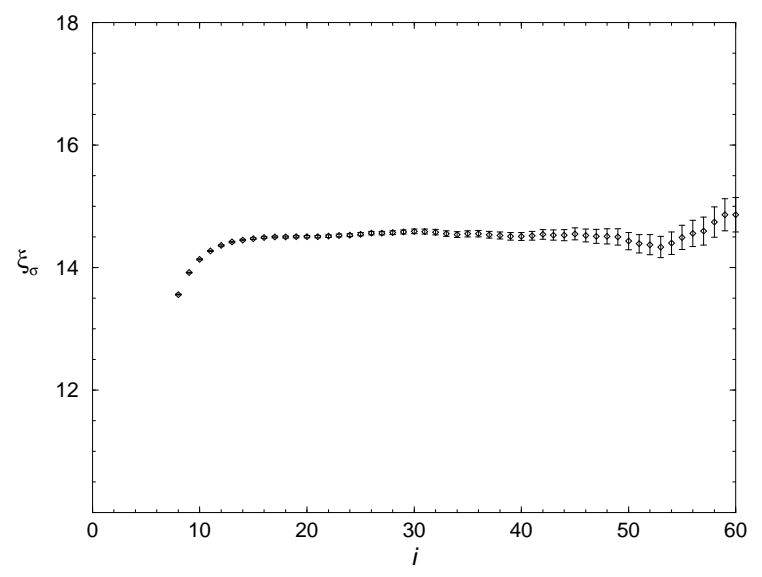

FIG. 1. Example of the set of estimators $\hat{\xi}_{i}$ for the magnetization density of a $18^{2} \times 214$ Ising system with periodic bc. The typical distance $\Delta$ in Eq. (6) was set to 8 .

The Ising model — Simulations of the Ising model were done at the most accurate estimate for the bulk inverse critical temperature available, $\beta_{c}=0.2216544(3)$ [17], where the influence of the given error in $\beta_{c}$ on the results for the correlation lengths was checked via a temperature reweighting technique and found negligible compared to the statistical errors; this applies to the other models considered in this note as well. To be able to perform a FSS analysis, simulations were done for system sizes between $4^{2} \times 48$ and $30^{2} \times 356 \approx 3 \times 10^{5}$ sites, accumulating about eight million independent measurements for each system.

As is obvious from the example in Fig. 2(a) the final estimates for the correlation lengths show up an almost perfect linear scaling behavior as a function of the transverse system size $L_{x}$. Considering the amplitudes $\hat{\xi} / L_{x}$ reveals, however, that corrections to the leading linear scaling behavior are relevant and can be clearly resolved within the accuracy of the data, cp. Fig. 2(b). In order to extract the leading amplitudes in the scaling regime nonlinear fits of the form

$$
\xi\left(L_{x}\right)=A L_{x}+B L_{x}^{\alpha}
$$

were done. Even though some field theoretical estimates for the correction exponents exist [8], we decided to keep $\alpha$ as a parameter, ending up at an effective correction exponent that takes higher order corrections into account, which have some importance for the small systems; successively dropping systems from the small $L_{x}$ end while monitoring the goodness of fit parameters $\chi^{2}$ and $Q$ then acts as a consistency check. As a rule, the overall corrections are negative for systems with periodic bc and 
positive in the case of antiperiodic bc.

As a result of this fitting procedure we arrive at the following final estimates for the amplitudes $A$ in Eq. (7) and their ratios:

$$
\begin{aligned}
& A_{\sigma}=0.8183(32) \\
& A_{\epsilon}=0.2232(16) \\
& A_{\sigma} / A_{\epsilon}=3.666(30) \\
& A_{\sigma}=0.23694(80) \\
& A_{\epsilon}=0.08661(31) \\
& A_{\sigma} / A_{\epsilon}=2.736(13)
\end{aligned}
$$

Comparing this to the ratio of scaling dimensions 11.,18, 19 ,

$$
x_{\epsilon} / x_{\sigma}=\frac{(1-\alpha) / \nu}{\beta / \nu}=\frac{2(\nu d-1)}{\nu d-\gamma}=2.7326(16),
$$

we find that the amplitude and exponent ratios agree very precisely in the case of antiperiodic bc across the torus, while in the periodic case they differ by an amount of some thirty sigma. In comparison to a first exploration by Weston [7], who found ratios of about 3.7 for periodic and 2.6 for antiperiodic bc, the precision could be increased by over an order of magnitude.
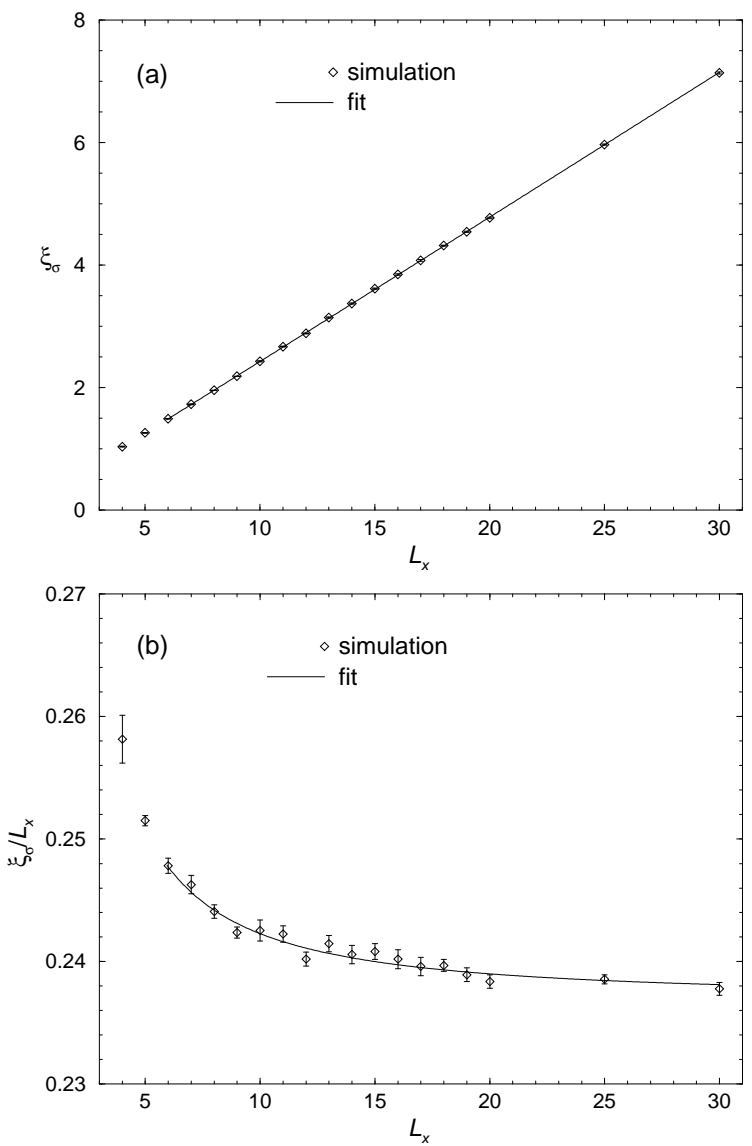

FIG. 2. (a) FSS plot for the spin correlation length $\xi_{\sigma}\left(L_{x}\right)$ of the 3D Ising model with antiperiodic bc. (b) Scaling of the amplitudes $\xi_{\sigma} / L_{x}$. Solid lines represent least-square fits according to Eq. (7).
$X Y$ and Heisenberg model - Although being stringent in itself, up to this point the above result is a singular, maybe casual, statement for the special case of the Ising model. Believing in a universal law needs a broader backing with successful examples, two of which being considered here.

Simulations for the XY and Heisenberg model were done at the estimated inverse critical temperature values $\beta_{c}=0.4541670(32)$ and $\beta_{c}=0.693004(7)$, respectively, which are weighted means of recent literature estimates [11,19, 21]. Investing about three years of workstation time for both models altogether and using the same system sizes as in the Ising case, we took between four and eighteen million independent measurements for each system, both for periodic and antiperiodic bc. Applying the outlined tools of data analysis we arrive at scaling and amplitude plots similar to those in Fig. 2. Traversing the above described fitting procedure leads to final estimates for the amplitudes $A_{\sigma}$ and $A_{\epsilon}$ according to Eq. (7), which are shown in Table [1. Comparing the results for the ratios $A_{\sigma} / A_{\epsilon}$ with the ratio $x_{\epsilon} / x_{\sigma}$ of scaling dimensions, we arrive at a highly precise agreement for the case of antiperiodic bc and an obvious divergence in the standard periodic bc situation for both, the XY and the Heisenberg model. Thus a linear relation between scaling amplitudes and scaling dimensions according to Eq. (11) is almost certainly valid for three generic, non-trivial examples of 3D spin models, and one might well assume, that it is satisfied for the whole class of $\mathrm{O}(n)$ spin models, a view which is supported by further simulations for the $n=10$ case 116 and an analytic result for the limiting case $n \rightarrow \infty$ [22], which is known to be equivalent to the spherical model 23]. In view of the analogous $2 \mathrm{D}$ results it is not too far fetched, then, to argue that the numerical results provide evidence that this relation might be of a universal, model independent kind.

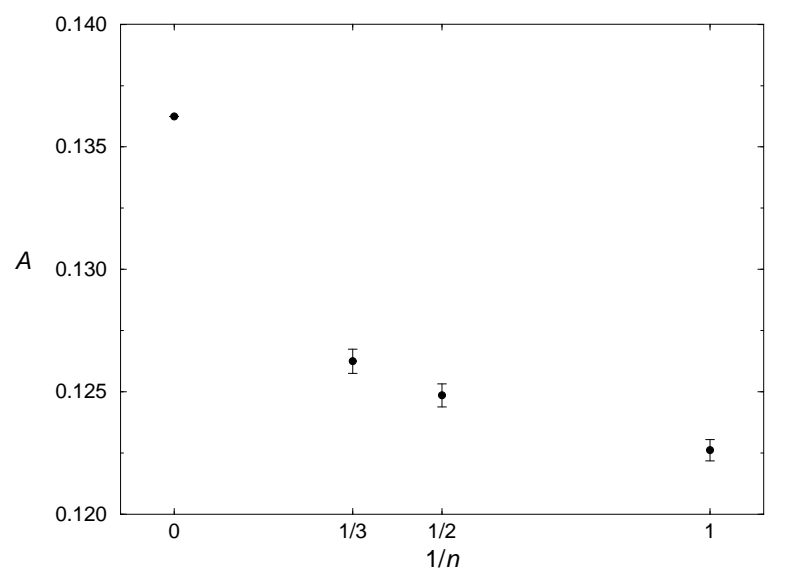

FIG. 3. Amplitudes $A$ according to Eq. (10) versus the inverse dimension of the order parameter $1 / n$ for $n=1,2,3, \infty$.

Universal amplitudes - Given the scaling amplitudes for the 3D systems with antiperiodic bc behave 
according to Eq. (17), one may ask further, what the amplitude $A$ in Eq. (11), that was $1 / 2 \pi$ in the $2 \mathrm{D}$ case, becomes in the 3D scenario and, furthermore, if it holds true that it is universal in the sense that all the modeldependent information is condensed in the scaling dimensions $x_{i}$. The transfer matrix approach cannot give an answer to this question, because in the Hamiltonian limit amplitudes are only given up to an overall normalization factor. Supposed such a relation holds, from the above results one can give an estimate for these amplitudes using the amplitudes $A_{\sigma}$, which are usually more accurate than $A_{\epsilon}$. Using the scaling dimensions of the spin of $x_{\sigma}=0.5175(5), x_{\sigma}=0.5178(15)$ and $x_{\sigma}=0.5161(17)$ for the Ising, the XY and the Heisenberg model, respectively, one has:

$$
A=A_{\sigma} x_{\sigma}=\left\{\begin{array}{ll}
0.12262(43) & \text { Ising } \\
0.12486(47) & \mathrm{XY} \\
0.12625(49) & \text { Heisenberg }
\end{array} .\right.
$$

Taking into account the corresponding amplitude of the spherical model, which is $A \approx 0.13624$ [22,24], and comparing the variation of these values with the given errors, as is shown in Fig. 3, it becomes clear that these amplitudes do in fact depend on the model under consideration and seem to vary smoothly and monotonically with the dimension $n$ of the order parameter.

To summarize, the amplitudes of the FSS of the correlation lengths of the magnetization and energy densities of $\mathrm{O}(n)$ spin models are linearly related to the corresponding scaling dimensions for the $T^{2} \times \mathbb{R}$ geometry when choosing antiperiodic instead of periodic bc across the torus; the amplitudes of this relation themselves depend, in contrast to the $2 \mathrm{D}$ case, on the model under consideration.

Note, however, that again in contrast to the $2 \mathrm{D}$ case, where the influence of boundary conditions on the operator content has been extensively explored [25], it is theoretically not understood up to now, why using antiperiodic bc in $3 \mathrm{D}$ should restore the $2 \mathrm{D}$ situation.

TABLE I. FSS amplitudes of the correlation lengths of the Ising, XY, and Heisenberg models on the $T^{2} \times \mathbb{R}$ geometry.

\begin{tabular}{clll}
\hline \hline model & & \multicolumn{1}{c}{$\mathrm{pbc}$} & apbc \\
\hline \multirow{4}{*}{ Ising } & $A_{\sigma}$ & $0.8183(32)$ & $0.23694(80)$ \\
& $A_{\epsilon}$ & $0.2232(16)$ & $0.08661(31)$ \\
& $A_{\sigma} / A_{\epsilon}$ & $3.666(30)$ & $2.736(13)$ \\
& $x_{\epsilon} / x_{\sigma}$ & \multicolumn{2}{c}{$2.7326(16)$} \\
\hline \multirow{4}{*}{$\mathrm{XY}$} & $A_{\sigma}$ & $0.75409(59)$ & $0.24113(57)$ \\
& $A_{\epsilon}$ & $0.1899(15)$ & $0.0823(13)$ \\
& $A_{\sigma} / A_{\epsilon}$ & $3.971(32)$ & $2.930(47)$ \\
\hline \multirow{4}{*}{ Heisenberg } & $x_{\epsilon} / x_{\sigma}$ & \multicolumn{2}{c}{$2.923(7)$} \\
& $A_{\sigma}$ & $0.72068(34)$ & $0.24462(51)$ \\
& $A_{\epsilon}$ & $0.16966(36)$ & $0.0793(20)$ \\
& $A_{\sigma} / A_{\epsilon}$ & $4.2478(92)$ & $3.085(78)$ \\
\hline \hline & $x_{\epsilon} / x_{\sigma}$ & \multicolumn{2}{c}{$3.091(8)$} \\
\hline
\end{tabular}

In view of the total lack of exact results for non-trivial $3 \mathrm{D}$ systems, it seems to us a rewarding challenge for the field theorists to explain these results.

We thank K. Binder for his constant and generous support. We are grateful to J. Cardy and M. Henkel for helpful discussions on the theoretical background. W.J. gratefully acknowledges support from the Deutsche Forschungsgemeinschaft through a Heisenberg Fellowship.

* Email: Martin.Weigel@itp.uni-leipzig.de

$\dagger$ Email: Wolfhard.Janke@itp.uni-leipzig.de

[1] J. L. Cardy, Conformal Invariance, in: Phase Transitions and Critical Phenomena, Vol. 11, eds. C. Domb and J. L. Lebowitz (Academic Press, London, 1987), p. 55.

[2] P. Christe, M. Henkel, Introduction to Conformal Invariance and Its Applications to Critical Phenomena (Springer, Berlin/Heidelberg/New York, 1993) [New Series m: Monographs, Lecture Notes in Physics, m 16].

[3] J. L. Cardy, J. Phys. A17, L385 (1984).

[4] J. L. Cardy, J. Phys. A18, L757 (1985).

[5] M. Henkel, J. Phys. A19, L247 (1986).

[6] M. Henkel, J. Phys. A20, L769 (1987).

[7] R. A. Weston, Phys. Lett. B248, 340 (1990).

[8] J. Zinn-Justin, Quantum Field Theory and Critical Phenomena (Clarendon Press, Oxford, 1996).

[9] U. Wolff, Phys. Rev. Lett. 62, 361 (1989).

[10] R. H. Swendsen, J.-S. Wang, Phys. Rev. Lett. 58, 86 (1987).

[11] W. Janke, Monte Carlo Simulations of Spin Systems, in: Computational Physics, ed. K.H. Hoffmann, M. Schreiber (Springer, Berlin, 1996), p. 10, and references therein.

[12] W. Janke, K. Nather, Phys. Rev. B48, 7419 (1993).

[13] M. Weigel, diploma thesis, Universität Mainz (1998), unpublished.

[14] B. Efron, The Jackknife, the Bootstrap and Other Resampling Plans, Society for Industrial and Applied Mathematics [SIAM], Philadelphia (1982).

[15] B. A. Berg, Double Jackknife Bias Corrected Estimators, Comp. Phys. Commun. 69, 7 (1992).

[16] M. Weigel, W. Janke, in preparation.

[17] A. L. Talapov, H. W. J. Blöte, J. Phys. A29, 5727 (1996).

[18] H. W. J. Blöte, E. Luijten, J. R. Heringa, J. Phys. A28, 6289 (1995).

[19] P. Butera, M. Comi, Phys. Rev. B56, 8212 (1997).

[20] H. G. Ballesteros, L. A. Fernández, V. Martín-Mayor, A. Muñoz Sudupe, Phys. Lett. B387, 125 (1996).

[21] A. P. Gottlob, M. Hasenbusch, J. Stat. Phys. 77, 919 (1994).

[22] M. Henkel, J. Phys. A21, L227 (1988); M. Henkel, R. A. Weston, J. Phys. A25, L207 (1992).

[23] H. E. Stanley, Phys. Rev. 176, 718 (1968).

[24] S. Allen, R. K. Pathria, J. Phys. A26, 5173 (1993).

[25] J. L. Cardy, Nucl. Phys. B275, 200 (1986). 